\title{
Sample Size Effect of Magnetomechanical Response for Magnetic Elastomers by Using Permanent Magnets
}

\author{
Tsubasa Oguro, ${ }^{1,2}$ Shuhei Sasaki, ${ }^{1,2}$ Yuri Tsujiei, ${ }^{1,2}$ Mika Kawai, ${ }^{1,2}$ Tetsu Mitsumata, \\ Tatsuo Kaneko, ${ }^{2,3}$ and Miklós Zrínyi ${ }^{3,4}$ \\ ${ }^{1}$ Graduate School of Science and Technology, Niigata University, Niigata 950-2181, Japan \\ ${ }^{2} J a p a n$ Science and Technology Agency, ALCA, Tokyo 102-0076, Japan \\ ${ }^{3}$ School of Materials Science, Japan Advanced Institute of Science and Technology (JAIST), Ishikawa 923-1292, Japan \\ ${ }^{4}$ Department of Biophysics and Radiation Biology, Laboratory of Nanochemistry, Faculty of Medicine, \\ Semmelweis University, Nagyvarad ter 4, Budapest 1089, Hungary \\ Correspondence should be addressed to Tetsu Mitsumata; tetsu@eng.niigata-u.ac.jp
}

Received 31 August 2016; Revised 5 December 2016; Accepted 12 December 2016; Published 2 January 2017

Academic Editor: Jean M. Greneche

Copyright (C) 2017 Tsubasa Oguro et al. This is an open access article distributed under the Creative Commons Attribution License, which permits unrestricted use, distribution, and reproduction in any medium, provided the original work is properly cited.

\begin{abstract}
The size effect of magnetomechanical response of chemically cross-linked disk shaped magnetic elastomers placed on a permanent magnet has been investigated by unidirectional compression tests. A cylindrical permanent magnet with a size of $35 \mathrm{~mm}$ in diameter and $15 \mathrm{~mm}$ in height was used to create the magnetic field. The magnetic field strength was approximately $420 \mathrm{mT}$ at the center of the upper surface of the magnet. The diameter of the magnetoelastic polymer disks was varied from $14 \mathrm{~mm}$ to $35 \mathrm{~mm}$, whereas the height was kept constant $(5 \mathrm{~mm})$ in the undeformed state. We have studied the influence of the disk diameter on the stress-strain behavior of the magnetoelastic in the presence and in the lack of magnetic field. It was found that the smallest magnetic elastomer with $14 \mathrm{~mm}$ diameter did not exhibit measurable magnetomechanical response due to magnetic field. On the opposite, the magnetic elastomers with diameters larger than $30 \mathrm{~mm}$ contracted in the direction parallel to the mechanical stress and largely elongated in the perpendicular direction. An explanation is put forward to interpret this size-dependent behavior by taking into account the nonuniform field distribution of magnetic field produced by the permanent magnet.
\end{abstract}

\section{Introduction}

Soft materials responsive to external stimuli such as temperature, $\mathrm{pH}$, and electric fields have attracted considerable attention as next generation actuators, devices with virtual reality, or soft robots, and so on. Magnetic elastomer is a soft material responsive to magnetic fields [1-8] and it is composed of flexible polymeric matrices and magnetic particles [9].

There are two methods to apply magnetic fields to magnetic elastomers. One is a method using an electromagnet where the spatial distribution of the magnetic field as well as its time dependence can be controlled. This is mainly used for theoretical investigations in homogeneous magnetic field [10]. Much less is known about the magnetoelastic behavior if the field is created by permanent magnets. In this case, one has to consider that the magnetic field around the permanent magnet is not homogeneous. The field strength on the surface varies and it is often significantly stronger at the edges. In the field of permanent magnet usually two effects, the magnetorheological effect (in uniform field) and magnetophoretic effect (in nonuniform field), may occur simultaneously. Thus the description of magnetoelastic effects is rather complicated task, taking into account the geometry and material properties of permanent magnet that determine the spatial distribution of magnetic field. However, the application of permanent magnet has various merits on practical use. Under the atmosphere with volatile organic substance, the use of electricity to drive the electromagnet is strictly limited. Magnetic field application by permanent magnets can be effective when power failure occurred due to, for example, disaster. A haptic device consisting of a permanent magnet and magnetic elastomers using permanent magnet can be fabricated mechanically and electrically simply because it 


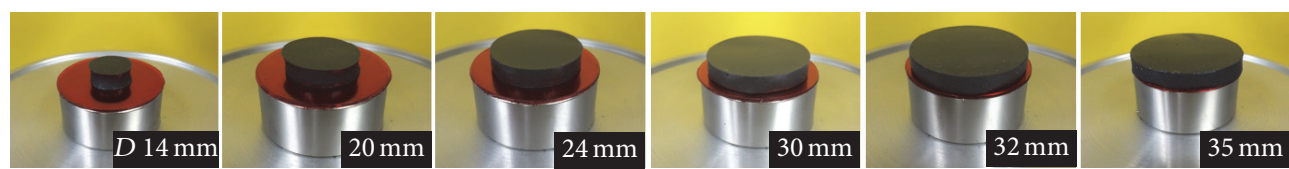

(a)

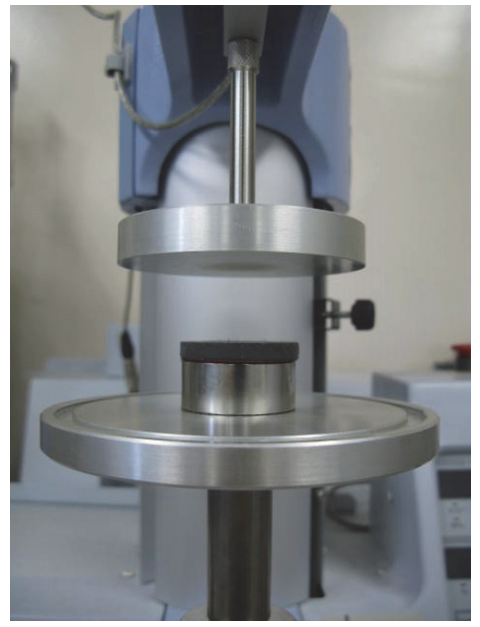

(b)

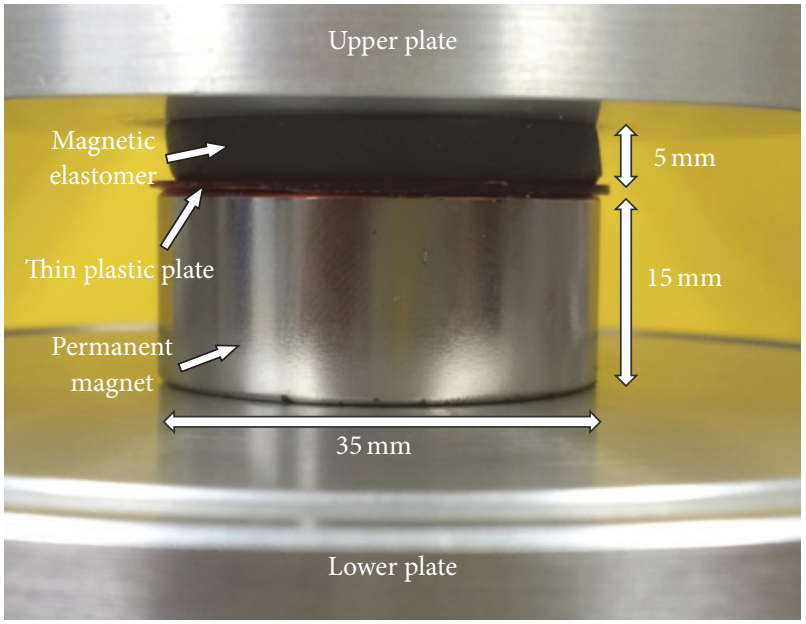

(c)

FIGURE 1: Photographs of (a) magnetic elastomers with various diameters (14 mm-35 mm) used in the present study. (b) Experimental setup for uniaxial compression apparatus in the present study. (c) Experimental setup for compression measurements.

does not need coils or electric power supplies. The difference using permanent magnet (nonhomogeneous field) and electromagnet (uniform field) demonstrated by our one of previous studies, where it was shown that the relative change in storage modulus exceeds 500 times using electromagnets; however only small change in Young's modulus with 2 3 times is observed for the measurement by using permanent magnets $[11,12]$. The main reason for the significant difference in magnetic response is due to inhomogeneity of the magnetic field produced by a permanent magnet.

The magnetic field produced by a permanent magnet is inhomogeneous; therefore, the size effect of magnetic elastomer as well as the geometry of magnetic elastomer and permanent magnet strongly affects the magnetic response. Under gradient fields, there arises a force proportional to the field gradient, and the force strongly affects the stress-strain behavior of bulk magnetic elastomers. In this study, we report the sample size effect on the magnetomechanical response for magnetic elastomers when a magnetic field was applied using a permanent magnet. We measured the stress-strain curves for magnetic elastomers with various diameters and the effect of sample size on the compressional stress is discussed.

\section{Experimental Procedure}

2.1. Synthesis of Magnetic Elastomers. A prepolymer method was used to synthesis magnetic elastomers. Polypropylene glycol $(\mathrm{Mw}=2000,3000)$, toluene diisocyanate (crosslinking agent), carbonyl iron (CI-CS) particles, and plasticizers (dioctyl phthalate, DOP) were mixed by a mechanical mixer for several minutes. The mixed liquid was poured in a silicon mold and cured on a hot stage for $60 \mathrm{~min}$ at $100^{\circ} \mathrm{C}$. The concentration of DOP was defined by the ratio of DOP to the matrix without magnetic particles and it was kept at $60 \mathrm{wt} . \%, \mathrm{DOP} /(\mathrm{DOP}+$ matrix $)$. The weight fraction of magnetic particles was kept at $28 \mathrm{wt} . \%, \mathrm{CI} / \mathrm{CI}$ + matrix), which corresponds to a volume fraction of 0.05 . The median diameter of the iron particles (BASF Co.) in dry state was measured to be $7.0 \pm 0.2 \mu \mathrm{m}$ using a particle size analyzer (SALD-2200, Shimadzu). We have prepared diskshaped magnetic elastomers with a height of $5 \mathrm{~mm}$ and the diameter of the identical samples was varied between $14 \mathrm{~mm}$ and $35 \mathrm{~mm}$. Figure 1(a) shows the photographs of magnetic elastomers with various diameters used in the present study.

2.2. Unidirectional Compression Measurements. The stressstrain curves for magnetic elastomers were obtained by a compression measurement at room temperature using a compression apparatus (EZ-SX, Shimadzu). The measurement was carried out at strains below 0.2 with a compression speed of $4 \mathrm{~mm} / \mathrm{min}$. A magnetic field was applied to magnetic elastomers using a permanent magnet (NeoMag Co. Ltd., Japan). The size of the permanent magnet was $15 \mathrm{~mm}$ thick and $35 \mathrm{~mm}$ in diameter as shown in Figure 1. The strength of the magnetic field was $420 \mathrm{mT}$ at the center of the magnet. Experimental setup for the uniaxial compression apparatus used in the present study is shown in Figure 1(b). As shown in Figure 1(c), a permanent magnet with a magnetic field of $420 \mathrm{mT}$ was set on the lower plate. A thin plastic plate with a thickness of $0.65 \mathrm{~mm}$ (red plate in the photo) was set on the permanent magnet in order to be sandwiched between the permanent magnet and magnetic elastomer. The apparent 
TABLE 1: Stress at $0 \mathrm{mT}$ and $420 \mathrm{mT}$ for magnetic elastomers with various diameters.

\begin{tabular}{lcc}
\hline$D(\mathrm{~mm})^{\mathrm{a}}$ & $0 \mathrm{mT}$ & $\sigma(\mathrm{kPa})^{\mathrm{b}}$ \\
\hline 14 & 36.6 & $420 \mathrm{mT}$ \\
20 & 47.5 & 39.6 \\
24 & 61.8 & 55.7 \\
30 & 61.2 & 78.2 \\
32 & 64.1 & 85.4 \\
35 & 88.3 & 103.0 \\
\hline
\end{tabular}

${ }^{\mathrm{a}}$ Sample diameter. ${ }^{\mathrm{b}}$ Stress at a strain of 0.2 .

stress was measured by compressing the magnetic elastomer with the upper plate. Magnetic elastomers with different diameter were put on the center of the permanent magnet having a diameter of $35 \mathrm{~mm}$ as shown in Figure 1. The stressstrain dependence for the samples in the presence and in the lack of magnetic field was determined at room temperature. The sample size effect of the magnetoelastomers is characterized by comparing the stress values at fixed strain of 0.2 .

\section{Results and Discussion}

Figure 2(a) shows the stress-strain curves for magnetic elastomers having a diameter of $14 \mathrm{~mm}$ and at field strength of $0 \mathrm{mT}$ and $420 \mathrm{mT}$, respectively. The measured stressstrain curves have shown a hardly observable difference seen by naked eyes, but the force transducer has indicated an increase from $36.6 \mathrm{kPa}$ to $39.6 \mathrm{kPa}$. In this case, the apparent change in the mechanical stress induced by the nonuniform magnetic field is extremely low. At some degree, larger diameter $(20 \mathrm{~mm})$ shown in Figure 2(b), the apparent stress has increased from $47.5 \mathrm{kPa}$ to $55.7 \mathrm{kPa}$ at the same strain. This increment is due to the magnetic interaction. Figures 2(c)-2(f) show that further enlargement of the radius of magnetoelastic gel disks results in gradually increasing increment of the magnetically induced strain. These results are summarized in Table 1 . It is seen in the table that the mechanical stress measured at 0.2 strain without magnetic field is not the same for the sample having identical chemical composition. The observed deviation may be due to nonhomogeneous spatial distribution of magnetic particles or particle aggregates which are locked in the polymer matrix. However, in all of the cases, one can see a definite increment in the stress induced by magnetic interactions. The magnitude of the magnetically induced stress increment denoted by $\Delta \sigma$ gradually increases with the sample size as shown by Figure 3 . This figure depicts the increment in the apparent stress due to the magnetic field at a strain of $0.2 \Delta \sigma$ as a function of the diameter of magnetic elastomers $D$. The value of $\Delta \sigma$ was determined by subtracting the off-field stress from on-field stress $(420 \mathrm{mT})$. For the largest disks, it results in a roughly 1.5-fold increase of stress compared with zero field. This finding has evidenced a clear magnetomechanical effect which should be taken into account when a magnetic elastomer is placed on a permanent magnet. The development of magnetic stress beside the mechanical stress is evidently a consequence of magnetic field distribution around the permanent magnet.

Figure 4 demonstrates the distribution of magnetic fields along the $x$ direction of the permanent magnet measured at the upper surface of permanent magnet. It is seen that the strength of magnetic field increased with the direction toward the fringe from the center of magnet. It is $418 \mathrm{mT}$ at the center and it increases to $448 \mathrm{mT}$ in both directions to the fringe. It must be mentioned that the magnetic field strength varies not only in the direction of $x$, but also in the perpendicular direction, $z$. Since the magnetic field shows strong nonuniform distribution in both directions (in plane $x$ and perpendicular direction above the upper surface, $z$ ) forces act on the magnetic particles, placed on the magnet. These forces are proportional to the magnetic field gradient. All of the magnetic particles are attracted to the higher field intensities. Since the magnetic particles are locked in the network of elastomer, therefore the attractive forces result in local deformation of the magnetic elastomer. There are at least two kinds of forces, one in the $x$ direction to the edges, which results in elongation to the edges, and the other perpendicular to the surface, $z$, causing contraction forward the surface of magnet. Due to the complexity of magnetic field distribution, both forces vary from point to point in the space, resulting in nonhomogeneous local deformation. In order to study the consequence of forces arising from nonuniform field on the shape of magnetic disks, we have compared the dependence of diameter, $L_{x}$ on the height, and $L_{z}$ of magnetic elastomers having different initial diameter.

Figure 5(a) shows the diameter dependence of the length ratio for magnetic elastomers with directions of $x$-axis $L_{x}$ and $z$-axis $L_{z}$ at a strain of 0.2 . The lengths of $L_{x}$ and $L_{z}$ were measured by image analysis. Figure 5(b) demonstrates the relationship between the length ratio $L_{x} / L_{z}$ and the diameter of magnetic elastomers $D$. Both of $L_{x} / L_{z}$ at $0 \mathrm{mT}$ and $420 \mathrm{mT}$ increased linearly with the diameter of magnetic elastomers. At diameters above $30 \mathrm{~mm}$, the values of $L_{x} / L_{z}$ at $420 \mathrm{mT}$ were clearly higher than those at $0 \mathrm{mT}$. This means that when the magnetic field was applied, the magnetic elastomers with diameters larger than $30 \mathrm{~mm}$ contracted in the direction to the surface and largely elongated in the perpendicular direction. Figure 5(c) shows the photographs representing the deformation for magnetic elastomers with diameters of $14 \mathrm{~mm}$ and $35 \mathrm{~mm}$. This figure provides evidence that the magnetic elastomer with a diameter of $35 \mathrm{~mm}$ largely elongated to the direction of edges and slightly compressed perpendicularly.

A schematic illustration representing a possible mechanism for the stress enhancement observed here is shown in Figure 6. The magnetic field strength of permanent magnets with a disk shape shows a maximum at the fringe. In particular, magnetic field strength is steeply weakened exceeding when the diameter of permanent magnet is larger than $35 \mathrm{~mm}$. Because of this, as a consequence, there arises a magnetic force $f_{x}$ originating from the magnetic field gradient along with the horizontal $x$-axis, which is proportional to $\mu \partial B_{x} / \partial x$ ( $\mu$ : magnetic permeability). The other perpendicular force $f_{z}$ proportional to $\mu \partial B_{z} / \partial z$ compressed 


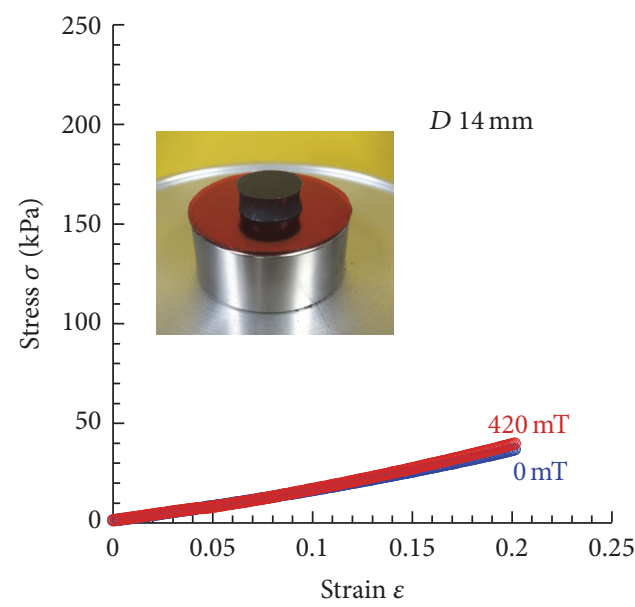

(a)

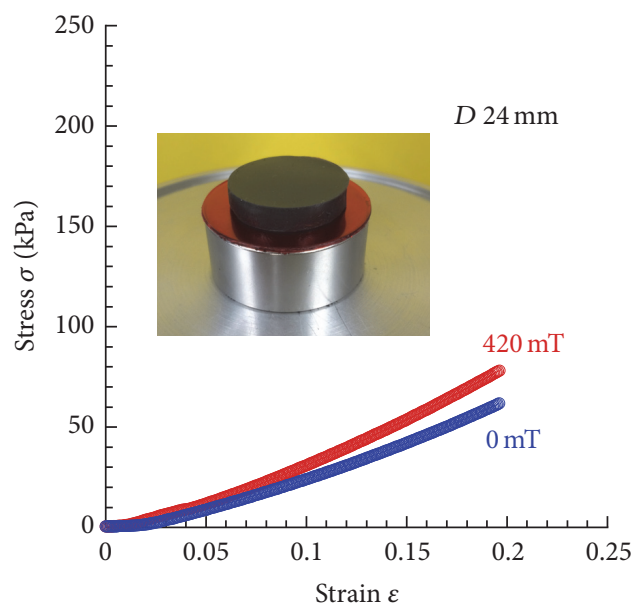

(c)

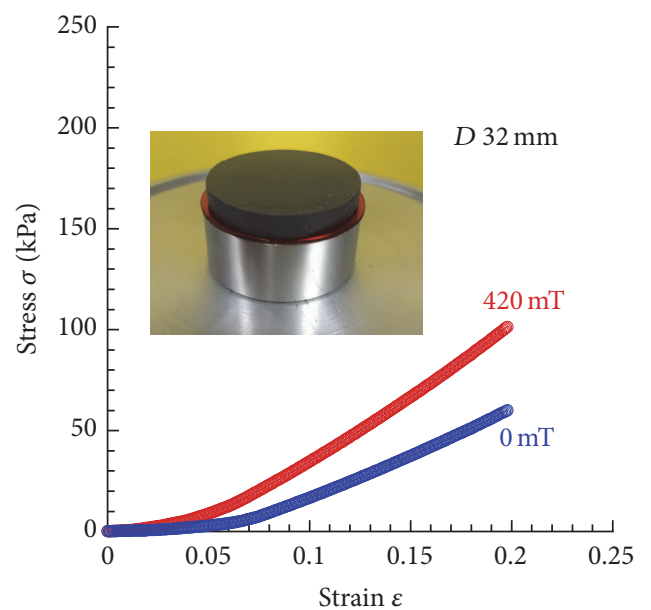

(e)

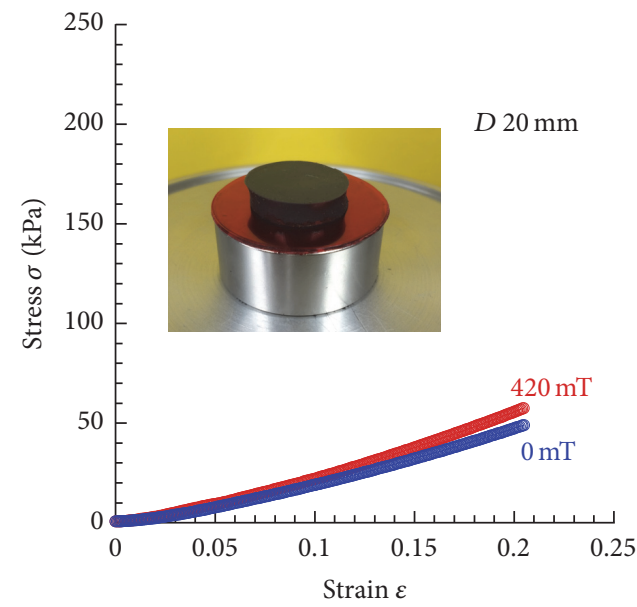

(b)

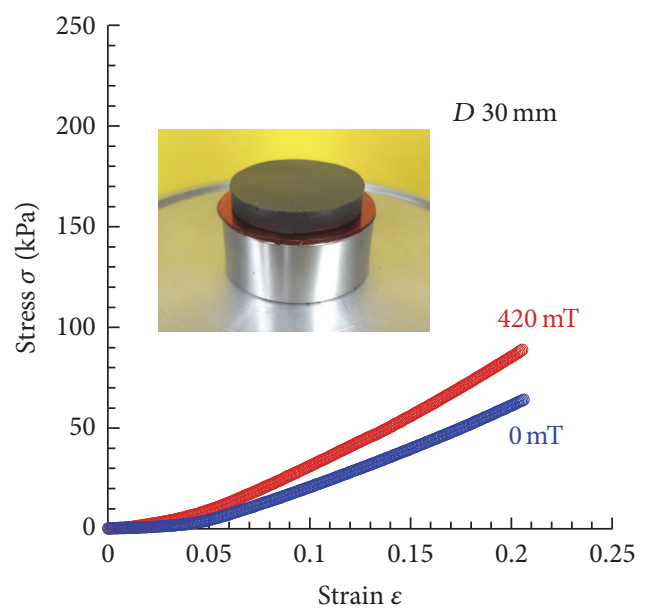

(d)

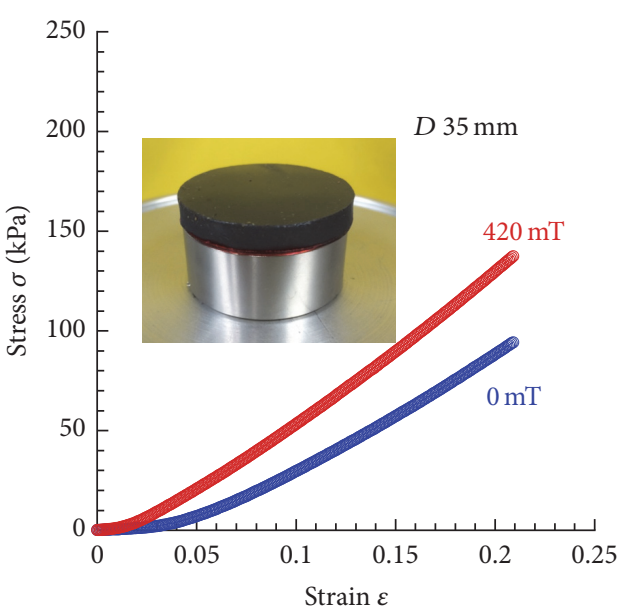

(f)

FIGURE 2: Stress-strain curves for magnetic elastomers with diameters of (a) $14 \mathrm{~mm}$ and (b) $35 \mathrm{~mm}$ when a permanent magnet with a magnetic field of $420 \mathrm{mT}$ was applied. 


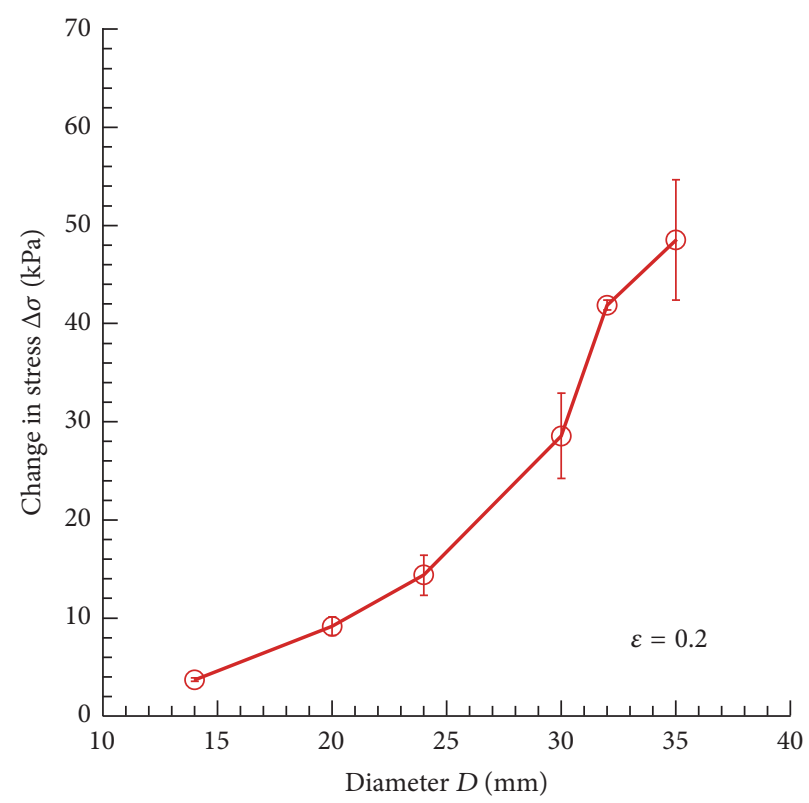

FIGURE 3: Change in apparent stress by magnetic fields at a strain of 0.2 as a function of the diameter of magnetic elastomers.

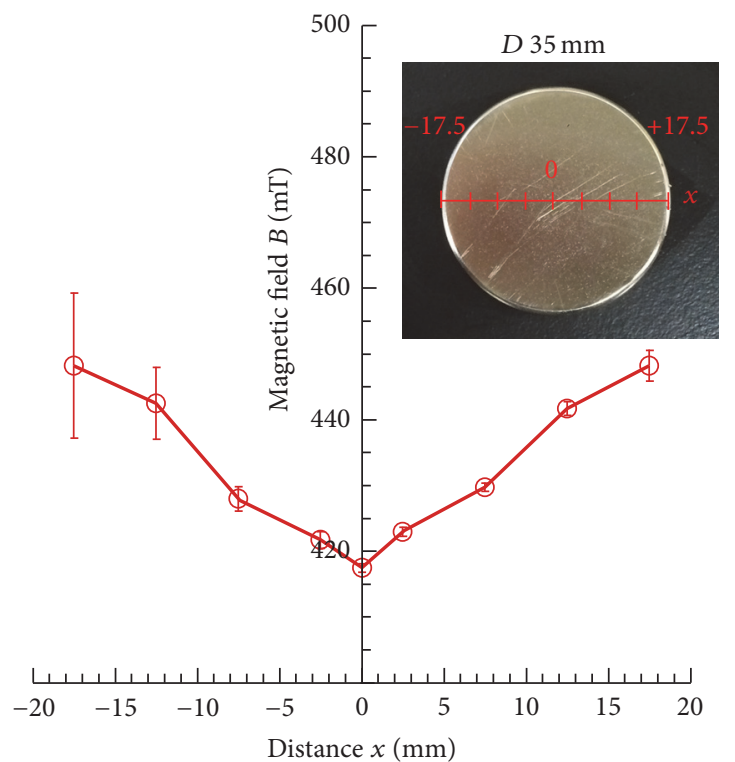

FIGURE 4: Magnetic field distribution along $x$-axis for permanent magnet used in the present study. Coordinate is indicated in the inset photo.

the sample to the surface, which also enhance the elongation in the $x$ direction to the edges. Therefore, the fact that strong magnetic force is generated at the fringe of permanent magnets can be considered. Magnetic elastomers around the fringe are pinned by the magnetic force, resulting in the depression of deformation of magnetic elastomers.

\section{Conclusions}

We have investigated the sample size effect on the magnetomechanical response when a magnetic field was applied by a permanent magnet. Unidirectional compression measurements exhibited that the magnetomechanical response by the permanent magnet was significantly weakened by decreasing the diameter of magnetic elastomers. On the other hand, it was found that the length ratio $L_{x} / L_{z}$ for magnetic elastomers exhibiting high magnetomechanical response took high values when the magnetic field was applied. This strongly indicates that magnetic elastomers under the magnetic field largely contracted in the direction parallel to the mechanical stress and largely elongated in the perpendicular direction when the magnetic field was applied. The large deformation 


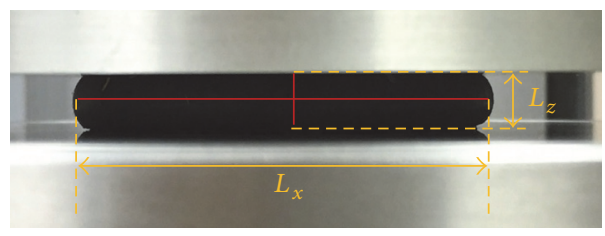

(a)
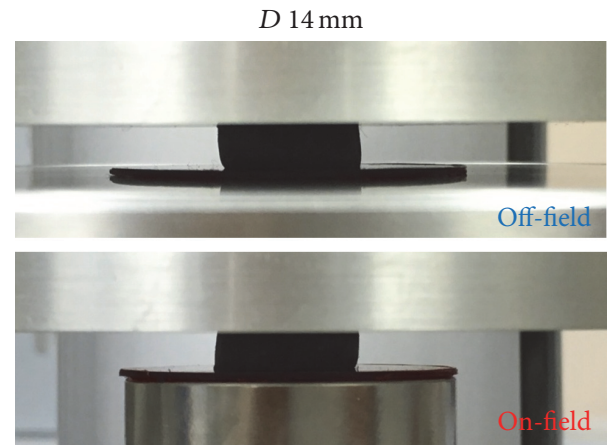

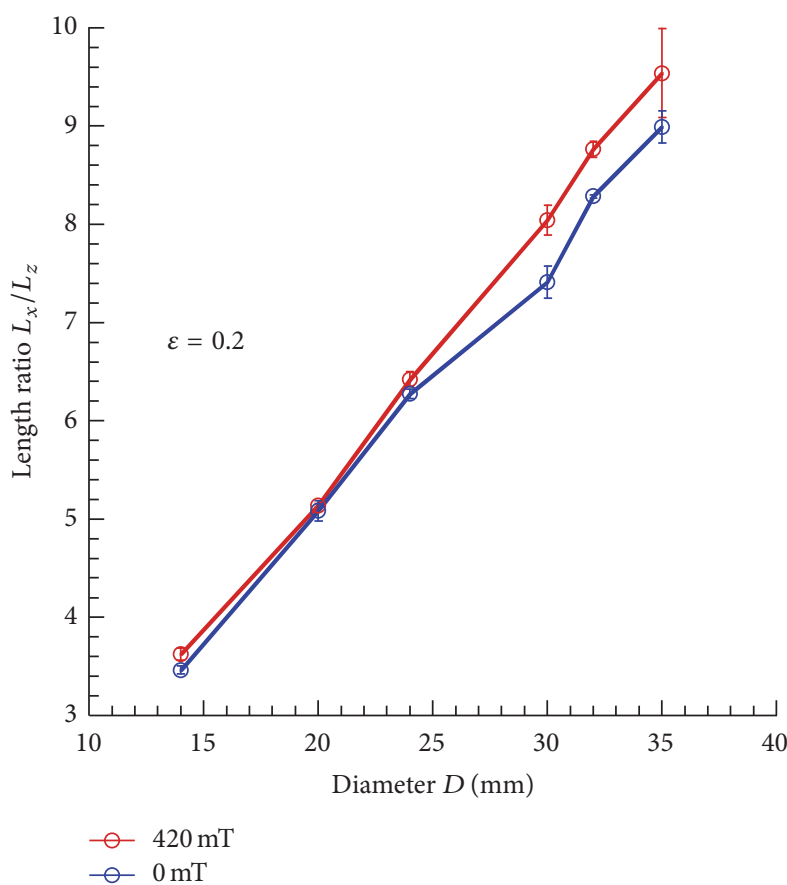

(b)

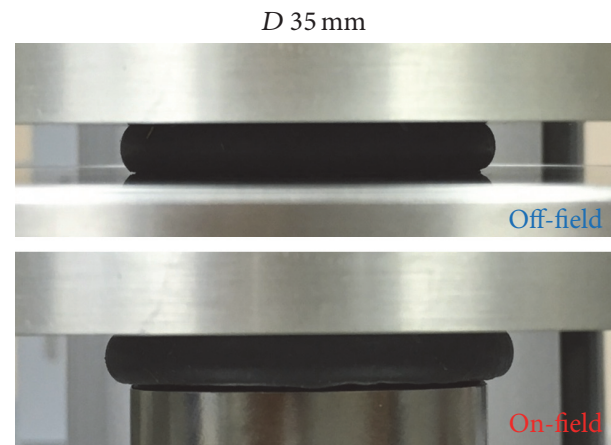

(c)

FIGURE 5: (a) Lengths of $L_{x}$ and $L_{z}$ at strain $\varepsilon=0.2$. (b) The length ratio $L_{x} / L_{z}$ as a function of diameter of magnetic elastomers. (c) Photographs for magnetic elastomers with diameters of $14 \mathrm{~mm}$ (left 2 photos) and $35 \mathrm{~mm}$ (right) with and without permanent magnet.

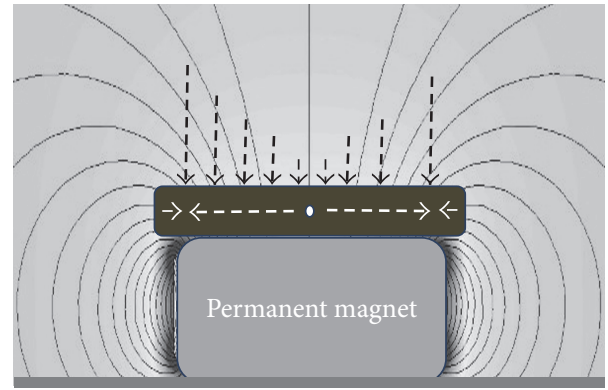

FIgURE 6: A schematic illustration representing a possible mechanism for the stress enhancement. The dashed arrows indicate the field gradient vectors that are responsible for local deformation.

observed here is considered to originate from the magnetic force due to the field gradient of the permanent magnet.
It was shown that a significant magnetomechanical effect occurs when a magnetic elastomer is placed on a permanent magnet. This finding has a significant impact on the practical application, despite the fact that the detailed description needs to be improved. In general, it is not easy to fabricate the devices of magnetic soft materials using electromagnets such as haptic devices because of the weakness of magnetic fields generated by the electromagnet. We firmly believe that the present results give us a guideline for fabricating devices consisting of magnetic soft materials driven by permanent magnets.

\section{Competing Interests}

The authors declare that none of them has any personal and financial relationship with people of any organization that can inappropriately influence his work as well as the conclusion drawn from this investigation. 


\section{Acknowledgments}

This research was partially supported by Sasaki Environment Technology Foundation, NAGAI N-S Promotions Foundation for Science of Perception, and UNION TOOL foundation. The authors are very grateful to Mr. W. Ito for his kind help to prepare this paper. Miklós Zrínyi acknowledges support from the Hungarian National Science Foundation (OTKA K 115259) and Japan Advanced Institute of Science and Technology. One of authors (Tetsu Mitsumata) is very grateful to Professor M. Zrinyi who initiated them to investigate magnetic gels 20 years ago, at the laboratory of Professors Y. Osada and J. P. Gong at Hokkaido University.

\section{References}

[1] Y. Xu, X. Gong, and S. Xuan, "Soft magnetorheological polymer gels with controllable rheological properties," Smart Materials and Structures, vol. 22, no. 7, Article ID 075029, 2013.

[2] X. Gong, Y. Xu, S. Xuan, C. Guo, L. Zong, and W. Jiang, “The investigation on the nonlinearity of plasticine-like magnetorheological material under oscillatory shear rheometry," Journal of Rheology, vol. 56, no. 6, pp. 1375-1391, 2012.

[3] Y. Xu, X. Gong, S. Xuan, X. Li, L. Qin, and W. Jiang, "Creep and recovery behaviors of magnetorheological plastomer and its magnetic-dependent properties," Soft Matter, vol. 8, no. 32, pp. 8483-8492, 2012.

[4] T. L. Sun, X. L. Gong, W. Q. Jiang, J. F. Li, Z. B. Xu, and W. $\mathrm{H}$. Li, "Study on the damping properties of magnetorheological elastomers based on cis-polybutadiene rubber," Polymer Testing, vol. 27, no. 4, pp. 520-526, 2008.

[5] A. V. Chertovich, G. V. Stepanov, E. Y. Kramarenko, and A. R. Khokhlov, "New composite elastomers with giant magnetic response," Macromolecular Materials and Engineering, vol. 295, no. 4, pp. 336-341, 2010.

[6] T. Mitsumata and S. Ohori, "Magnetic polyurethane elastomers with wide range modulation of elasticity," Polymer Chemistry, vol. 2, no. 5, pp. 1063-1067, 2011.

[7] T. Mitsumata, A. Honda, H. Kanazawa, and M. Kawai, "Magnetically tunable elasticity for magnetic hydrogels consisting of carrageenan and carbonyl iron particles," The Journal of Physical Chemistry B, vol. 116, no. 40, pp. 12341-12348, 2012.

[8] T. Mitsumata, S. Ohori, N. Chiba, and M. Kawai, "Enhancement of magnetoelastic behavior of bimodal magnetic elastomers by stress transfer via nonmagnetic particles," Soft Matter, vol. 9, no. 42, pp. 10108-10116, 2013.

[9] T. Mitsumata, K. Ikeda, J. P. Gong, Y. Osada, D. Szabó, and M. Zrínyi, "Magnetism and compressive modulus of magnetic fluid containing gels," Journal of Applied Physics, vol. 85, no. 12, pp. 8451-8455, 1999.

[10] M. Zrínyi, "Magnetoelasticity," in Magnetorheology, N. M. Wereley, Ed., chapter 3, pp. 56-74, RSC Publishing, Cambridge, UK, 2014.

[11] K. Negami and T. Mitsumata, "Flexible fluid pump using magnetic composite gels," e-Polymers, vol. 34, 2011.

[12] K. Nagashima, S. Kanauchi, M. Kawai, T. Mitsumata, S. Tamesue, and T. Yamauchi, "Nonmagnetic particles enhance magnetoelastic response of magnetic elastomers," Journal of Applied Physics, vol. 118, no. 2, Article ID 024903, 2015. 

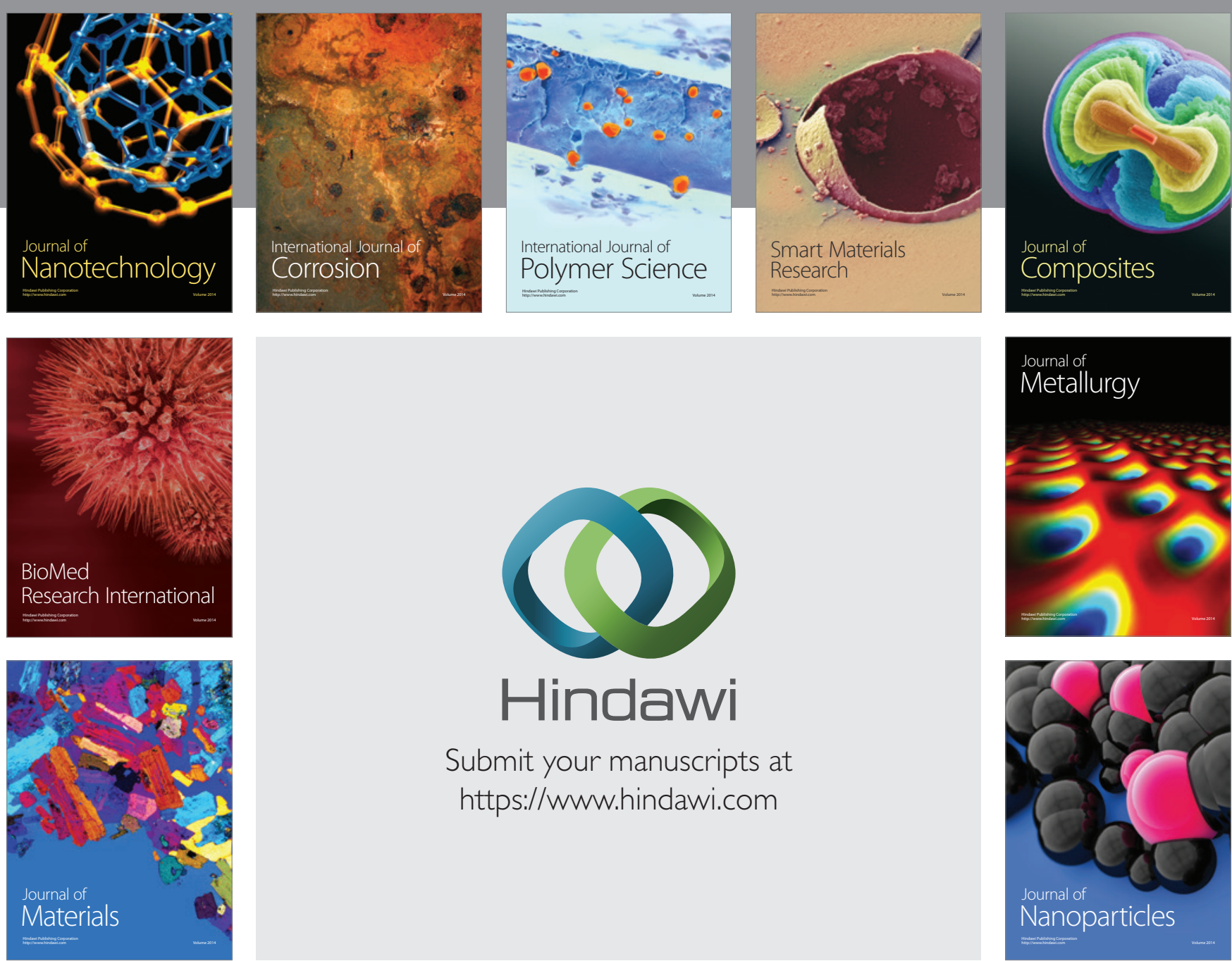

\section{Hindawi}

Submit your manuscripts at

https://www.hindawi.com

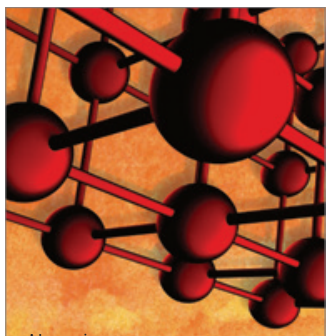

Materials Science and Engineering
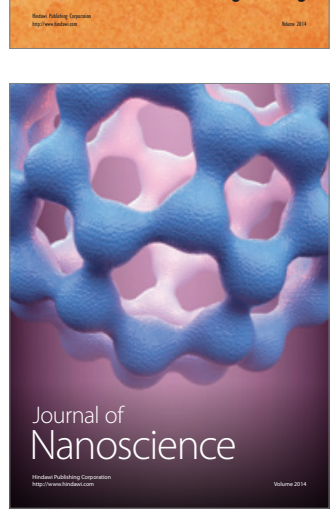
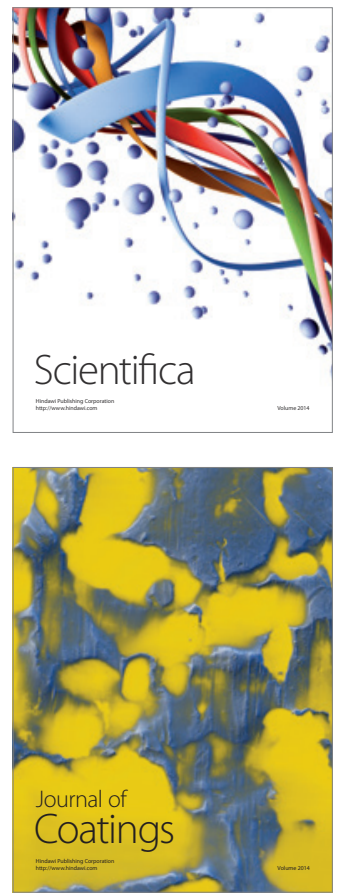
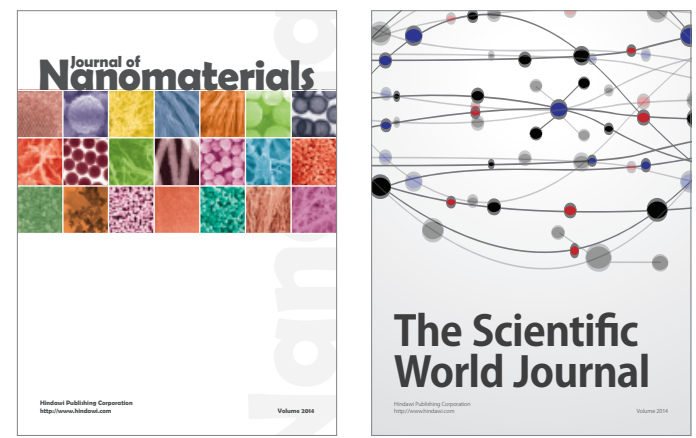

The Scientific World Journal
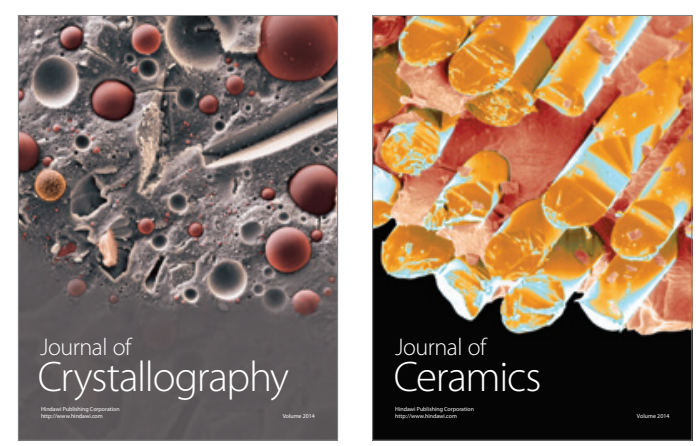
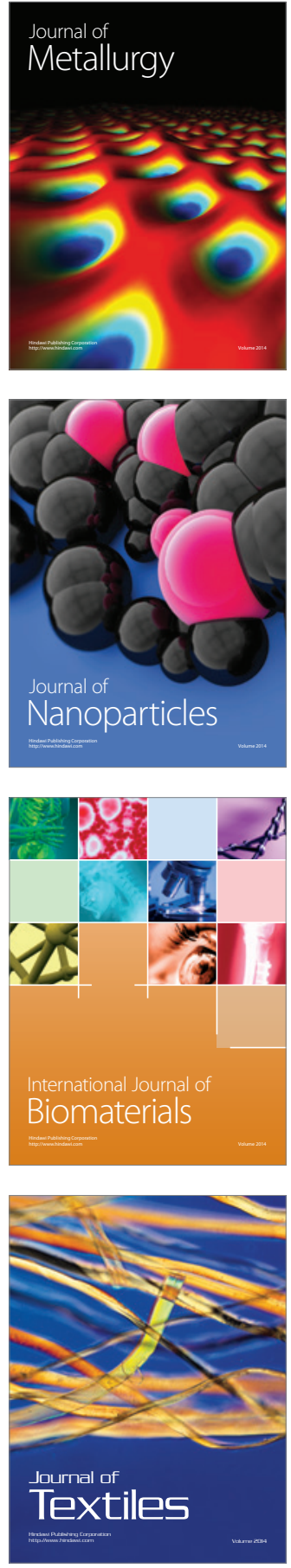